\title{
The Integrating Application of Machine learning and Internet of Things (IoT)
}

\author{
Yanda Chen \\ International School, Beijing University of Posts and Telecommunications, Beijing 100876, China;
}

MAME2013@163.com

Keywords: Machine learning, Big data; Internet of Things; application.

\begin{abstract}
At present, the new generation of information and communication technology, represented by Internet of Things, big data and cloud computing, is developing rapidly. Internet of things technology is accelerating to intelligent medical, new energy, materials and other areas of penetration through the continuous integration with other new technologies.Machine learning concludes lots of approaches (e.g. Deep learning, GMM) for different tasks like prediction, classification, recognition etc. The common point of those approaches is that they are all data-driven. How to make data more efficient, real-time, dynamic and large-scale is what we should pay attention to.This study explores the relationship between machine learning and the Internet of Things, as well as the significance of the integration of these two technologies. Accordingly, the paper expatiates on the application of big data in the Internet of Things industry. Finally, we explored the big data technology framework in Internet of Things. The integration of big data and Internet of Things is bound to bring the intelligence of human society to a new height. Its development prospects cannot be limited.
\end{abstract}

\section{Introduction}

The multilayer neural network model (deep learning) has strong learning ability to approximate any function. Making it combined with the big data, will obtain better description of the internal logic of big data information. Of course, even based on the traditional machine learning model, when we give the model more data, it is often able to get better results in less data. Most of the characteristics of the project are lossy. And most of the depth of learning to avoid this step. This determines the difference between traditional machine learning methods and depth learning models.

With the continuous development of the Internet of Things industry, in order to achieve "objects linked" and "people connected with objects", hundreds of millions of devices which can sense the connection of things, such as RFID, GPS, search engines, browsers and so on, are embedded in the physical equipment for data collection. Due to the increasing number of sensing devices, the massive data collected by the Internet of Things were in a blowout. In addition, cloud computing and other big data processing technology is widely used to achieve data analysis and continuous optimization of information transmission and exchange. Thus making the application needs of the Internet of Things industry in the field of intelligent identification, positioning, tracking, monitoring, management and other fields improve from conceptualization to commercial materialization [1].

Massive data are continually generated from a variety of Internet of Things sensing devices and application systems, and they will continue to grow in more and more complex and diversified ways. The complexity and format diversification of big data determine the diversity of service scenarios and types for big data in the Internet of Things service platform, which requires the Internet of Things service platform must be integrated big data technology to respond [2]. Based on this, this paper discusses the relationship between big data and Internet of Things, and explains the significance of big data for the Internet of things and the application of big data in the Internet of Things. Finally, we explored the big data technology framework in the Internet of Things. 


\section{The Relationship between Big Data and Internet of Things}

\subsection{Internet of Things Gave Birth to Big Data.}

As an important extension of the Internet, the Internet of Things is widely interconnected by building a perceptual infrastructure in a pervasive environment. It builds an information ecosystem composed of networks, information and services between the human world, the physical world and the information world [3]. With the rapid development of the Internet of Things, a variety of cross-industry and interdisciplinary sensing devices and terminals can quickly access the network and get together. A large number of sensors in the Internet of Things convert all kinds of information in the material world into electrical signals and transmits them to the upper application system through various types of networks. It is foreseeable that a trend has been formed, that is, massive unstructured data will grow rapidly. The growth of these data is not linear, however, as more and more sensors are being developed, manufactured and put into production, the amount of data will show exponential growth. This trend is unstoppable.

\subsection{Big Data Enriched the Applications of Internet of Things.}

The Internet of things under the age of big data makes the value of big data to be reflected. In the application of Internet of Things technology, useful data can be mined out to form useful information to create value through collecting big data by means of constructing the intelligent building, digital medical, remote sensing, intelligent transportation, environmental monitoring and protection and others, and analyzing data by cloud computing. For example, the world's top car company is using a remote sensing technology to effectively identify the identity of the owner to avoid the theft of luxury cars. The technology can effectively remember and identify the driver's driving position to determine whether the driver is driving in concentration, and effectively prevent the occurrence of traffic accidents. The technology remembers the weight of the owner, the normal driving force and the relevant key indicators to identify the owner of the identity through installing a sensor under the car seat. Once the driver does not match the original data, car anti-theft system will automatically identify the danger, and automatically feed the information back to the owner through the wireless technology, so that owners can make the appropriate way to deal with.

\subsection{The Significance of Big Data for Internet of Things.}

Internet of things usually includes the perceptual layer, the network layer and the application layer. The perceptual layer generates massive data. The application layer reprocesses the data of the perceptual layer. It mines the user's behavior and preferences through intelligent processing and analyzing massive data generated by the perceptual layer, finds products and services more in line with user interests and habits from the messy data, and adjusts and optimizes targeted products and services. Thus providing business applications that meet the needs of different users, and these applications are the core business value of the Internet of Things. In short, the Internet of Things produce big data, and big data promote the Internet of Things. In this sense, the core of the Internet of Things industry is the extensive use of big data analysis means for intelligent management and optimization of operations. From the perspective of commercial and industrial development, big data behind the Internet of Things can provide information from business support to business decision-making, having the essence of commercial applications, which can speed up the process of business applications of things networking. The development and application of big data analysis technology make various internet of things applications more colorful.

\section{The Application of Big Data in the Internet of Things Industry}

At present, the applications of big data in the Internet of Things industry mainly include the following four levels, data collection, data transfer, and data processing and data applications. The four levels of the Internet of Things industry each play a different role. Among them, data collection and data transfer are the basis of big data in the Internet of Things industry application. And data processing and data application is the core of the application of big data in the Internet of Things 
industry. The following content of the application of big data in the Internet of Things Industry conducted a focus on the discussion.

\subsection{Data Collection.}

Data collection in the Internet of Things has always played an important role, that is, the data collection is the base of big data application in the Internet of Things industry. Only when the data is collected, can the data be analyzed and processed. With the development and progress of science and technology, Internet of things in the data collection technology is also in the state of the continuous development and innovation. At present, data collection tools used in the industry of the Internet of things are sensors, bar code, WEB2.0, RFID and mobile terminal processing technology and so on. The development and innovation of the data collection tools make the data types obtained by the Internet industry workers show a variety of characteristics. If we can analyze the types of data collection, we can find that the collected data information not only contains the physical data information, but also some information about the geographical location. Therefore, the information can be seen in a very wide range. Data collection seems not that simple. In general, data collection also involves data de-noising process and information extraction. The data of Internet of things is diverse and non-structural. In general, the data of the Internet of Things has a certain degree of noise. So how to remove the noise to extract valuable information is often the key to the Internet of things information extraction. In the Internet of Things industry, the relevant staff in Internet of Things, out of the convenience of information transportation and information processing, often make the corresponding de-noising process in the initial stage of the data collection. In addition, the Internet of things must be some load in the process of running. In order to reduce the load during operation, we also need to extract the important data.

\subsection{Data Storage.}

With the continuous improvement of economic level, the amount of data the Internet involved in is also increasing every day. In order to handle the data involved in the Internet of Things efficiently and timely, the data must be stored. With the development of economic, the data storage technology in the Internet of things continues to improve. At present, the most popular data storage technology is undoubtedly the distributed storage technology of the non-relational database. The data storage technology is proposed by Google. The specific operation method is the use of large-scale low-cost server to deal with the corresponding non-relational data. Long-term development practice shows that distributed storage technology of the non-relational database promotes the development of the Internet of things industry to a large extent. Following the non-relational database distributed storage technology, there have appeared the cloud storage, distributed file system and other new data storage in the Internet of Things industry. From the deep level of analysis, these new storage methods draw on the mobile Internet technology to a large extent, and thus make some data and information stored anywhere, anytime, as well as analyzed and processed, which will virtually promote the speed of the Internet of things.

\subsection{Data Analysis.}

Data analysis also plays an important role in the Internet of Things industry. The data mining, the prediction of the data model and the presentation of the data results in the Internet of Things industry are all part of the data analysis. It can be seen that the status of data analysis in the Internet of Things is of vital importance. To some extent, it seems that the fact that big data is regarded as the core part of the application in the Internet of Things industry is reflected in the data analysis process. The Internet of Things industry staff are often based on the existing data, analyzing and processing. This thus plays a predictive role for the data. After the data analysis, some enterprise decision-makers will change the marketing strategy to make the appropriate adjustments maximize the benefits. The existing data analysis methods are SVM in statistics, Naive Bayes in taxonomy and other analytical methods. And the data analysis tools commonly used is such as Mahout of Hadoop and so on. Data analysis in the current Internet of things industry is a very important content. Through data analysis, on the one hand the enterprises can predict the next business strategy, making the development of enterprises in a positive direction. On the other hand, through the analysis and process of the data, the 
enterprises can understand the users' habits and preferences further, and then lay the foundation for providing users with better service.

\section{Big Data Technology Framework in Internet of Things}

Collecting a large number of data needs a strong basic framework, which is not only used to support data storage and query, but also need to support the extraction of effective data. In order to meet the requirements of implementing a variety of unique needs, the data needs various types of learning functions. For example, it is one of the most common requirements to build an aggregation model by analyzing historical data [4]. However, considering that data may be stored in different networks, we think it may be a very complicated process. Hadoop is a distributed storage and processing framework that meets the needs of data analysis in the situation of the Internet of Things. Over the past few years, Hadoop has evolved into an ecosystem that encompasses a variety of applications and services, such as supporting efficient data storage, organization, retrieval (including queries), search, processing, and resource scheduling and maintenance and many other core functions [5].

\section{Conclusion}

Nowadays, the Internet of Things is faced with the strategic development opportunities and challenges of the era of big data. This paper discusses the relationship between big data and Internet of Things, and focuses on the application of big data in the Internet of Things, and discusses the big data technology framework in Internet of Things. The handshake of Internet of things and big data not only will make things used more widely, but also extend the long value industrial chain based on the big data. Therefore, if the concept of big data development is instilled into the whole development process of the Internet of Things, it will promote the process that the development of the Internet of Things drives big data development. Besides, the applications of big data will accelerate the pace of the Internet of Things development.

\section{References}

[1]. Aggarwal, C. C., Ashish, N., \& Sheth, A. (2013). The internet of things: A survey from the data-centric perspective. In Managing and mining sensor data (pp. 383-428). Springer US.

[2]. Da Xu, L., He, W., \& Li, S. (2014). Internet of things in industries: A survey.IEEE Transactions on industrial informatics, 10(4), 2233-2243.

[3]. Stankovic, J. A. (2014). Research directions for the internet of things. IEEE Internet of Things Journal, 1(1), 3-9.

[4]. Botta, A., De Donato, W., Persico, V., \& Pescapé, A. (2014, August). On the integration of cloud computing and internet of things. In Future Internet of Things and Cloud (FiCloud), 2014 International Conference on (pp. 23-30). IEEE.

[5]. Botta, A., De Donato, W., Persico, V., \& Pescapé, A. (2016). Integration of cloud computing and internet of things: a survey. Future Generation Computer Systems, 56, 684-700. 